\title{
NATURAL DUALITIES FOR DIHEDRAL VARIETIES
}

\author{
B. A. DAVEY and R. W. QUACKENBUSH
}

(Received 3 August 1994; revised 19 December 1994)

Communicated by L. Kovács

\begin{abstract}
A strong, natural duality is established for the variety generated by a dihedral group of order $2 \mathrm{~m}$ with $\mathrm{m}$ odd. This is the first natural duality for a non-abelian variety of groups.

1991 Mathematics subject classification (Amer. Math. Soc.): primary 08A02; secondary 08B05. Keywords and phrases: duality, natural duality, strong duality, group, dihedral group, variety.
\end{abstract}

\section{Introduction}

Which finitely generated quasivarieties of groups admit a natural duality? The main theorem of this paper (Theorem 2) extends the list of known examples into the nonabelian realm.

For the benefit of readers not familiar with the theory of natural dualities, we begin with a brief review of what is meant by 'admitting a natural duality' and refer to Davey [4] or the forthcoming text Clark and Davey [3] for a detailed account.

Let $\underline{\mathbf{M}}$ be a finite group and let $\underset{\sim}{\mathbf{M}}=\langle M ; G, H, R, \mathscr{T}\rangle$ be a topological structure on the same underlying set, where

(a) each $g \in G$ is a homomorphism $g: \underline{\mathbf{M}}^{n} \rightarrow \underline{\mathbf{M}}$ for some $n \in \mathbb{N} \cup\{0\}$,

(b) each $h \in H$ is a homomorphism $h: \operatorname{dom}(h) \rightarrow \underline{\mathbf{M}}$ where $\operatorname{dom}(h)$ is a subgroup of $\underline{\mathbf{M}}^{n}$ for some $n \in \mathbb{N}$,

(c) each $r \in R$ is (the universe of) a subalgebra of $\underline{\mathbf{M}}^{n}$ for some $n \in \mathbb{N}$,

(d) $\mathscr{T}$ is the discrete topology.

Whenever (a), (b) and (c) hold, we say that the operations in $G$, the partial operations in $H$ and the relations in $R$ are algebraic over $\underline{\mathbf{M}}$. These compatibility conditions

The research of the second author was supported by a grant from the NSERC of Canada.

(C) 1996 Australian Mathematical Society $0263-6115 / 96 \$ A 2.00+0.00$ 
between the structure on $\underline{\mathbf{M}}$ and the structure on $\underset{\sim}{\mathbf{M}}$ guarantee that there is a naturally defined dual adjunction between the quasivariety $\mathscr{A}:=\mathbb{U S P} \underline{\mathbf{M}}$ generated by $\underline{\mathbf{M}}$ and the topological quasivariety $\mathscr{X}:=\mathbb{A} \mathbb{S}_{\mathrm{c}} \mathbb{P} \mathbf{M}$ generated by $\mathbf{M}$. For all $\mathbf{A} \in \mathscr{A}$ the homset $D(\mathbf{A}):=\mathscr{A}(\mathbf{A}, \underline{\mathbf{M}})$ is a closed substructure of the direct power $\widetilde{\mathbf{M}}^{A}$ and for all $\mathbf{X} \in \mathscr{X}$ the homset $E(\mathbf{X}):=\mathscr{X}(\mathbf{X}, \underset{\sim}{\mathbf{M}})$ is a subgroup of the direct power $\underline{\mathbf{M}}^{X}$. It follows easily that the contravariant hom-functors $\mathscr{A}(-, \underline{\mathbf{M}}): \mathscr{A} \rightarrow \mathscr{S}$ and $\mathscr{X}(-, \underset{\sim}{\mathbf{M}}): \mathscr{X} \rightarrow \mathscr{S}$, where $\mathscr{S}$ is the category of sets, lift to contravariant functors $D: \mathscr{A} \rightarrow \mathscr{X}$ and $E: \mathscr{X} \rightarrow \mathscr{A}$. For each $\mathbf{A} \in \mathscr{A}$ there is a natural embedding $e_{\mathrm{A}}$ of A into $E D(\mathbf{A})$ given by evaluation: for each $a \in A$ and each $x \in D(\mathbf{A})=\mathscr{A}(\mathbf{A}, \underline{\mathbf{M}})$ define $e_{\mathrm{A}}(a)(x):=x(a)$. Similarly, for each $\mathbf{X} \in \mathscr{X}$ there is an embedding $\epsilon_{\mathbf{X}}$ of $\mathbf{X}$ into $D E(\mathbf{X})$. A simple calculation shows that $e: \operatorname{id}_{\mathscr{A}} \rightarrow D E$ and $\epsilon: \operatorname{id}_{\mathscr{X}} \rightarrow D E$ are natural transformations. If $e_{\mathbf{A}}$ is an isomorphism for all $\mathbf{A} \in \mathscr{A}$ we say that $\mathbf{M}$ yields a (natural) duality on $\mathscr{A}$. If, moreover, $\epsilon_{\mathbf{X}}$ is an isomorphism for all $\mathbf{X} \in \mathscr{X}$, we say that $\mathbf{M}$ yields a full duality on $\mathscr{X}$ (in which case $\mathscr{A}$ and $\mathscr{X}$ are dually equivalent categories). If there is some choice of $G, H$ and $R$ such that $\mathbf{M}$ yields a duality on $\mathscr{A}$ then we say that $\underline{\mathbf{M}}$ (or $\mathscr{A}$ ) admits a natural duality or, more colloquially, is dualizable.

The best known examples of dualizable groups are the finite cyclic groups: if $\underline{\mathbf{C}}_{m}=\left\langle C_{m} ; \cdot,{ }^{-1}, 1\right\rangle$ is an $m$-element cyclic group, then $\mathbf{C}_{m}:=\left\langle C_{m} ; \cdot,^{-1}, 1, \mathscr{T}\right\rangle$ yields a full duality on $\mathscr{A}_{m}:=\mathbb{I S P} \underline{C}_{m}$. (In this case both $H$ and $R$ are empty.) The class $\mathscr{A}_{m}$ is the variety of abelian groups of exponent $m$ while $\mathscr{X}_{m}:=\mathbb{U} \mathbb{S}_{\mathrm{c}} \mathbb{P} \mathbf{M}$ is the category of compact (totally disconnected) topological abelian groups of exponent $m$.

We shall refer to this as the Pontryagin Duality on $\mathscr{A}_{m}$ as it can be obtained by restricting the Pontryagin duality for the class of all abelian groups to the subvariety $\mathscr{A}_{m}$. The general theory of natural dualities affords several simple, direct proofs of this duality which avoid the application of Pontryagin's sledgehammer-see Davey and Werner [6] or Clark and Davey [1]. In fact, every finite abelian group $\mathbf{M}$ is dualizable: it is shown in Davey [5] that if $G=\left\{\cdot,{ }^{-1}, 1\right\} \cup$ End $\underline{\mathbf{M}}$ and $H=R=\emptyset$, then $\underset{\sim}{\mathbf{M}}$ yields a duality on $\mathscr{A}:=\mathbb{\triangle S P} \underline{\mathrm{M}}$ which will not in general be full.

Thus, this paper is a contribution to the solution of the following fundamental problem.

PROBLEM. Which finite groups admit a natural duality?

The general theory of natural dualities tells us that it order to show that $\underset{\sim}{\mathbf{M}}$ yields a full duality on $\mathscr{A}$ it is sufficient to prove the following three conditions-

(CLO) for each $n \in \mathbb{N}$, every morphism $t:{\underset{\sim}{\mathbf{M}}}^{n} \rightarrow \underset{\sim}{\mathbf{M}}$ is an n-ary term function on $\underline{\mathbf{M}}$,

(INJ) $\underset{\sim}{\mathbf{M}}$ is injective in $\mathscr{X}$,

(STR) for every non-empty set I, for each substructure $\mathbf{X}$ of $\mathbf{M}^{l}$ and for each 
$\boldsymbol{y} \in M^{I} \backslash X$ there exist morphisms $\varphi, \psi:{\underset{\sim}{\mathbf{M}}}^{I} \rightarrow \underset{\sim}{\mathbf{M}}$ such that $\left.\varphi\right|_{\mathbf{X}}=\left.\psi\right|_{\mathbf{X}}$ but $\varphi(\boldsymbol{y}) \neq \psi(\boldsymbol{y})$.

Together, (CLO) and (INJ) guarantee that $\underset{\sim}{\mathbf{M}}$ yields a duality on $\mathscr{A}$. The condition (CLO) is of independent algebraic interest as it asserts that the $n$-ary term functions on $\underline{\mathbf{M}}$ are precisely the maps $t: M^{n} \rightarrow M$ which preserve the operations in $G$, partial operations in $H$ and relations in $R$. A duality which satisfies (STR) is called a strong duality. By Clark and Davey [1], a duality is strong if and only if it is full and (INJ) holds. Every known full duality is strong. Note that in the case of the variety $\mathscr{A}_{m}$, we may always choose the morphism $\varphi$ in the condition (STR) to be the constant map onto 1 , whence (STR) is equivalent in this case to

if $\mathbf{X}$ is a closed subgroup of $\mathbf{C}_{m}^{l}$ and $\boldsymbol{y} \in C_{m}^{l} \backslash X$, then there is a continuous homomorphism $\psi:{\underset{\mathbf{C}}{m}}_{m}^{I} \rightarrow{\underset{\mathbf{C}}{m}}_{m}$ such that $\left.\psi\right|_{\mathbf{X}}=\underline{\mathbf{1}}$ while $\psi(\boldsymbol{y}) \neq 1$, where $\underline{\mathbf{1}}$ is the constant map onto 1 .

The conditions (INJ) and (STR) can both be reduced to the finite case (thus elimimating all topological considerations) whenever $H$ is empty and $R$ is finite, as in the case of the finite cyclic group. The results of Clark and Davey [3] show that if $\mathbf{M}$ yields a strong duality on $\mathscr{A}$ and $\underline{\mathbf{M}}$ is not injective in $\mathscr{A}$, then the set $H$ of partial operations must be non-empty. Since the dihedral group $\underline{\mathbf{D}}_{m}$ is not injective in the quasivariety it generates, if we wish to obtain a strong duality for the quasivariety generated by $\mathbf{D}_{m}$ we will have no choice but to include partial operations in the type of $\underset{\sim}{\mathbf{D}_{m}}$.

\section{The dihedral groups}

Let $\underline{\mathbf{D}}_{m}=\left\langle D_{m} ; \cdot\right\rangle$ be the dihedral group of order $2 m$ presented by $a^{m}=b^{2}=1$ and $b a=a^{m-1} b$. In the case that $m$ is odd, we will establish a strong duality for $\mathscr{A}:=\mathbb{\triangle S} \mathbb{P} \underline{\mathbf{D}}_{m}$, the quasivariety generated by $\underline{\mathbf{D}}_{m}$ (in this case, $0 \mathbb{S P} \underline{\mathbf{D}}_{m}$ is actually the variety generated by $\underline{\mathbf{D}}_{m}$ ). Hence, we now assume that $m$ is odd. The dual category will be the topological quasivariety $\mathscr{X}:=\square \mathbb{S}_{c} \mathbb{P} \mathbb{\sim}_{m}$ where

$$
\stackrel{\mathbf{D}}{m}_{m}=\left\langle D_{m} ; \alpha, 1 ;+, * ; \mathscr{T}\right\rangle .
$$

As usual, the topology, $\mathscr{T}$, is discrete. The total operations are the automorphism $\alpha$ of $\underline{\mathbf{D}}_{m}$, given by $\alpha(a)=a$ and $\alpha(b)=a b$, and the nullary operation 1 . The first partial operation, + , is simply the partial binary map from $\underline{\mathbf{D}}_{m}^{2}$ to $\underline{\mathbf{D}}_{m}$ which is the restriction to $H:=\{1, b\}$ of the group operation on $\underline{\mathbf{D}}_{m}$ (thus, $b^{i}+b^{j}:=b^{i+j}$ ). The second partial operation is slightly more complicated. Let $\epsilon$ be the (unique) retraction of $\underline{\mathbf{D}}_{m}$ onto $\mathbf{H}$. Let $N \subseteq D_{m}$ be the kernel of $\epsilon$ in the group theoretic sense (thus, $\left.N=\left\{1, a, a^{2}, \ldots, a^{m-1}\right\}\right)$, and let $K \subseteq D_{m}^{2}$ be the kernel of $\epsilon$ in the general-algebraic 
sense (thus,

$$
\begin{aligned}
K & :=\left\{(u, v) \in D_{m}^{2} \mid \epsilon(u)=\epsilon(v)\right\}=N \times N \cup N b \times N b \\
& =\left\{\left(a^{i} b^{k}, a^{j} b^{k}\right) \mid 0 \leqslant i, j \leqslant m-1 \text { and } 0 \leqslant k \leqslant 1\right\}
\end{aligned}
$$

is the congruence corresponding to $N$ ). Define $*$ to be the partial map from $\underline{\mathbf{D}}_{m}^{2}$ to $\underline{\mathbf{D}}_{m}$ whose domain is $K$ with $a^{i} b^{k} * a^{j} b^{k}:=a^{i+j} b^{k}$. Note that on $N$ the operation $*$ is just the original group operation while on $N b$ the operation $*$ is the translation of the original group operation on $N$. Thus, $\langle N b ; *\rangle$ is a group and right translation by $b$ is a group isomorphism from $\langle N ; \cdot\rangle$ onto $\langle N b ; *\rangle$. Observe that $\alpha$ is the identity map on $N$ and is the cycle $\left(b, a b, a^{2} b, \ldots, a^{m-1} b\right)$ on the other coset $N b$. In the case that $m$ is odd, $\alpha$ is an inner automorphism; indeed, if $m=2 k+1$, then $\alpha(g)=a^{-k} g a^{k}$ for all $g \in D_{m}$. Each of these operations and partial operations is algebraic over $\underline{\mathbf{D}}_{m}$. This is obvious in each case except $*$. That $*$ is algebraic follows from the lemma below.

LEMMA 1. Let $\boldsymbol{G}$ be a group and let $\epsilon$ be a retraction of $\boldsymbol{G}$ onto a subgroup $\mathbf{H}$. Let $N$ be the kernel of $\epsilon$ and let

$K:=\left\{(u, v) \in G^{2} \mid \epsilon(u)=\epsilon(v)\right\}=\bigcup\left\{\epsilon^{-1}(h) \mid h \in H\right\}=\bigcup\{N h \times N h \mid h \in H\}$

be the congruence corresponding to $N$. Define a partial binary operation $*$, with domain $K$, by $x h * y h:=x y h$ for all $x, y \in N$ and $h \in H$, or equivalently, define $u * v:=u \epsilon(u)^{-1} v=u \epsilon(v)^{-1} v$ for all $(u, v) \in K$.

(a) (The restriction of) $*$ is a well-defined group operation on $N h$ for each $h \in H$. Moreover, right translation by $h$ is an isomorphism of $\langle N ; \cdot\rangle$ onto $\langle N h ; *\rangle$.

(b) The partial operation * is associative wherever it is defined. It will be commutative wherever it is defined provided $N$ is abelian.

(c) The map * :K $\rightarrow G$ is a homomorphism if and only if $N$ is abelian.

PROOF. For (a) we need only that $N$ is a subgroup and that $H$ is a class of representatives for the right cosets of $N$. A trivial calculation establishes (b).

For (c) we need to know that $\boldsymbol{K}$ is a subgroup of $\underline{\mathbf{D}}_{m}^{2}$ (that is, that $N$ is a normal subgroup) and that, for all $h, k \in H$, the representative of the right coset $N h k$ is $h k$, that is, that $\mathbf{H}$ is a subgroup of $\boldsymbol{G}$. Together this says precisely that $\epsilon$ is a retraction onto the subgroup $\mathbf{H}$.

We wish to prove that $*$ is a homomorphism, that is,

(1) $(\forall w, x, y, z \in N)(\forall h, k \in H)(w h \cdot y k) *(x h \cdot z k)=(w h * x h) \cdot(y k * z k)$,

if and only if $N$ is abelian. Let $w, x, y, z \in N$ and $h, k \in H$; then since $N$ is normal, there exist $y^{\prime}, z^{\prime} \in N$ such that $h y=y^{\prime} h$ and $h z=z^{\prime} h$. Hence

$$
(w h \cdot y k) *(x h \cdot z k)=\left(w y^{\prime} h k\right) *\left(x z^{\prime} h k\right)=\left(w y^{\prime} x z^{\prime}\right) h k
$$


and

$$
(w h * x h) \cdot(y k * z k)=w x h y z k=w x y^{\prime} h z k=\left(w x y^{\prime} z^{\prime}\right) h k .
$$

Thus (1) holds provided $N$ is abelian, and choosing $h=k=w=z=1$ in (1) shows that (1) implies that $N$ is abelian.

We can now state the main result of this paper.

THEOREM 2. The structure $\mathbf{D}_{m}$ yields a strong duality on $\mathscr{A}$, that is, the homfunctors $D: \mathscr{A} \rightarrow \mathscr{X}$ and $E: \mathscr{X} \rightarrow \mathscr{A}$ give a dual category equivalence between $\mathscr{A}:=\mathbb{U S P \mathbb { P }}$ and $\mathscr{X}:=\mathbb{U} \mathbb{S}_{\mathrm{c}} \mathbb{P} \underline{\mathbf{D}}_{m}$ and $\underline{\mathbf{D}}_{m}$ is injective in $\mathscr{X}$.

Unfortunately, we do not have an axiomatization of the class $\mathscr{X}$.

If $\mathbf{D}_{m}$ yields a strong duality on $\mathscr{A}$, then every closed substructure $\mathbf{X}$ of a power of $\underline{\mathbf{D}}_{m}$ must (at least) be closed under every endomorphism of $\underline{\mathbf{D}}_{m}$ and moreover every $\mathscr{X}$-morphism from $\mathbf{X}$ to $\mathbf{D}_{m}$ must preserve the actions of the endomorphisms of $\underline{\mathbf{D}}_{m}$ on $\mathbf{X}$ (see Clark and Davey [1]). We begin our proof of Theorem 2 by establishing this necessary condition plus a little more. The partial operation $*$ induces a partial operation on $D(\mathbf{A})$ for all $\mathbf{A} \in \mathscr{A}$ and in particular on $D\left(\underline{\mathbf{D}}_{m}\right)=$ End $\underline{\mathbf{D}}_{m}$ : if $e, f \in$ End $\underline{\mathbf{D}}_{m}$, then $e * f$ is defined if and only if for each $u \in G$ either $e(u), f(u) \in N$ or $e(u), f(u) \in N b$. Denote the constant endomorphism by 1 .

\section{LEMMA 3. Assume that $m$ is odd.}

(a) For all $k, l \in \mathbb{Z}_{m}$, there is an endomorphism $e$ of $\underline{\mathbf{D}}_{m}$ such that $e(a)=a^{k}$ and $e(b)=a^{l} b$. Moreover, every non-constant endomorphism of $\underline{\mathbf{D}}_{m}$ is of this form. Thus $\mid$ End $\underline{\mathbf{D}}_{m} \mid=m^{2}+1$.

(b) $\underline{1} * \underline{1}=\underline{1}$ and, for all $e \in$ End $\underline{\mathbf{D}}_{m}$, the product $e * \underline{1}$ exists if and only if $e=\underline{1}$. If $e, f \in$ End $_{m}$ with $e \neq \underline{1}$ and $f \neq \underline{1}$, then $e * f$ exists.

(c) $\left\langle\right.$ End $\left.\underline{\mathbf{D}}_{m} \backslash\{\underline{1}\} ; *\right\rangle$ is an abelian group isomorphic to $\mathbb{Z}_{m}^{2}$ and is generated (as a group) by the powers (with respect to composition of maps) of the automorphism $\alpha$. The retraction $\epsilon$ is an identity element for $*$ on End $\underline{\mathbf{D}}_{m} \backslash\{1\}$.

PROOF. It is easily seen that $a_{1}:=a^{k}$ and $b_{1}:=a^{l} b$ satisfy the defining relations for $\underline{\mathbf{D}}_{m}$ and hence $e(a):=a^{k}$ and $e(b):=a^{l} b$ does determine an endomorphism of $\underline{\mathbf{D}}_{m}$. We must now show that when $m$ is odd, there are no other non-constant endomorphisms. Let $e$ be an endomorphism of $\underline{\mathbf{D}}_{m}$ and define $a_{1}:=e(a)$ and $b_{1}=e(b)$. Thus

$$
a_{1}^{m}=1, \quad b_{1}^{2}=1 \quad \text { and } \quad b_{1} a_{1}=a_{1}^{m-1} b_{1} .
$$

As $m$ is odd, we must have $a_{1} \in N$ as every element of $N b$ has order 2: thus $a_{1}=a^{k}$ for some $k \in \mathbb{Z}_{m}$. If $b_{1} \in N$, then since $N$ contains no elements of order 2 , we have 
$e(b)=b_{1}=1$. In this case (2) implies that $k=0$ and consequently $e=\underline{1}$. If $b_{1} \in N b$, then $e(b)=a^{l} b$ for some $l \in \mathbb{Z}_{m}$, as required. Hence (a) holds.

It is clear that $\underline{1} * \underline{1}$ exists and equals $\underline{1}$. If $e \in$ End $\underline{\mathbf{D}}_{m}$ with $e \neq \underline{1}$, then by (a) we have $e(b) \in N b$. Hence

$$
(\underline{1}(b), e(b))=(1, e(b)) \notin K=N^{2} \cup N b^{2}=\operatorname{dom}(*)
$$

and consequently $1 * e$ is not defined on $D\left(\underline{\mathbf{D}}_{m}\right)=$ End $\underline{\mathbf{D}}_{m}$. If $e, f \in$ End $\underline{\mathbf{D}}_{m} \backslash\{\underline{1}\}$, then $e(N) \subseteq N, e(N b) \subseteq N b, f(N) \subseteq N$ and $f(N b) \subseteq N b$ and hence, for all $u \in D_{n}$,

$$
(e(u), f(u)) \in K=N^{2} \cup N b^{2}=\operatorname{dom}(*),
$$

that is, $e * f$ is defined on $D\left(\underline{\mathbf{D}}_{m}\right)=$ End $\underline{\mathbf{D}}_{m}$. Thus (b) holds.

Since, by Lemma 1 , the partial operation $*$ on $D_{n}$ is commutative and associative wherever it is defined, it follows that $\left\langle\right.$ End $\left.\underline{\mathbf{D}}_{m} \backslash\{\underline{1}\} ; *\right\rangle$ is a commutative semigroup. It is easily seen that the map $\gamma:$ End $\underline{\mathbf{D}}_{m} \rightarrow \mathbb{Z}_{m}^{2}$, given by $\gamma(e)=(k, l)$ if $e(a)=a^{k}$ and $e(b)=a^{l} b$, is an isomorphism. It is clear from the definition of $*$ that $\epsilon$ is an identity element for $*$ on End $\underline{\mathbf{D}}_{m}$. Denote powers of $e \in$ End $\underline{\mathbf{D}}_{m}$ with respect to composition of maps by $e^{s}$ and powers with respect to $*$ by $e^{[s]}$. A simple calculation shows that $e:=\mathrm{id}_{\underline{\mathbf{D}}_{m}}^{[m-1]} * \alpha^{l} * \mathrm{id}_{\underline{\mathbf{D}}_{m}}^{[k]}$ satisfies $e(a)=a^{k}$ and $e(b)=a^{l} b$, whence the set $\left\{\alpha^{s} \mid s=1, \ldots, m\right\}$ generates the group $\left\langle\right.$ End $\left.\underline{\mathbf{D}}_{m} \backslash\{\underline{1}\} ; *\right\rangle$. This proves (c).

We are now ready to prove Theorem 2 . We will establish this strong duality by proving the conditions (INJ), (CLO) and (STR)—see Propositions 4, 5 and 8 below. The first is of independent, group-theoretic interest.

PROPOSITION 4. Let $m$ be odd. A map $\varphi: D_{m}^{n} \rightarrow D_{m}$ is a term function on the dihedral group $\underline{\mathbf{D}}_{m}$ if and only if $\varphi$ preserves the action of the automorphism $\alpha$, the constant 1 and the partial operations + and $*$.

PROOF. As Kovács observes in [7], since $\mathscr{A}$ is the product variety $\mathscr{A}_{m} \mathscr{A}_{2}$, Corollary 21.13 of Neumann [8] implies that the $n$-generated free group in $\mathscr{A}$ is an extension of a $F_{\mathscr{A}_{m}}(k)$ by $F_{\mathscr{A}_{2}}(n)$, where $k=(n-1)\left|F_{\mathscr{A}_{2}}(n)\right|+1$. Thus the $n$-generated free group over $\underline{\mathbf{D}}_{m}$ has cardinality $2^{n} m^{(n-1) 2^{n}+1}$. Since the (partial) operations on $\underline{\mathbf{D}}_{m}$ are algebraic over $\underline{\mathbf{D}}_{m}$, every $n$-ary $\underline{\mathbf{D}}_{m}$-term function belongs to $\mathscr{X}\left(\mathbf{D}_{m}^{n}, \mathbf{D}_{m}\right)$. Thus it suffices to show that $\left|\mathscr{X}\left(\mathbf{D}_{m}^{n}, \mathbf{D}_{m}\right)\right| \leqslant 2^{n} m^{(n-1) 2^{n}+1}$.

Let $\varphi \in \mathscr{X}\left(\underline{\mathbf{D}}_{m}^{n}, \mathbf{D}_{m}\right)$. Since + is the original group operation on the subgroup $\{1, b\}$ of $\underline{\mathbf{D}}_{m}$, and since $\varphi$ preserves + , the restriction $\left.\varphi\right|_{\{1, b\}^{n}}$ is an abelian group homomorphism. There are exactly $2^{n}$ such homomorphisms. We will show that each homomorphism from $\{1, b\}^{n}$ to $\{1, b\}$ has at most $m^{(n-1) 2^{n}+1}$ extensions to a member of 
$\mathscr{X}\left(\mathbf{D}_{m}^{n}, \mathbf{D}_{m}\right)$. Recall our retraction $\epsilon$ of $D_{m}$ onto $H=\{1, b\}$; on $D_{m}^{n}$ it is a retraction onto $\{1, b\}^{n}$. Now recall our partial operation $*$; it turns each of the two cosets of $N$ into an abelian group isomorphic to $\mathbb{Z}_{m}$. On $D_{m}^{n}$ it turns each of the cosets of $N^{n}$ into an abelian group isomorphic to $\mathbb{Z}_{m}^{n}$. But each such coset is equal to $\epsilon^{-1}(\boldsymbol{h})$ for some $\boldsymbol{h} \in\{1, b\}^{n}$. Since $\varphi$ preserves $*$, there are at most $m^{n}$ possibilities for $\left.\varphi\right|_{\epsilon^{-1}(\boldsymbol{h})}$. (Note that $\varphi\left(\epsilon^{-1}(\boldsymbol{h})\right)$ will be contained in either $N$ or $N b$, depending on whether $\varphi(\boldsymbol{h})$ equals 1 or $b$ ). This yields $\left|\mathscr{X}\left(\mathbf{D}_{m}^{n}, \mathbf{D}_{m}\right)\right| \leqslant 2^{n} m^{n 2^{n}}$, since for each of the $2^{n}$ choices for $\boldsymbol{h} \in\{1, b\}^{n}$ we have at most $m^{n}$ choices for $\left.\varphi\right|_{\epsilon^{-1}(h)}$. We now take into account the effect of the automorphism $\alpha$. If $\boldsymbol{h} \neq \boldsymbol{1}$, then $\alpha(\boldsymbol{h}) \neq \boldsymbol{h}$, but $\epsilon(\alpha(\boldsymbol{h}))=\boldsymbol{h}$ whence $\alpha(\boldsymbol{h}) \in \epsilon^{-1}(\boldsymbol{h})$. As $\varphi(\alpha(\boldsymbol{h}))$ is determined by $\varphi(\boldsymbol{h})$ (since $\varphi$ preserves $\alpha$ ) and as $\alpha(\boldsymbol{h})$ is an element of order $m$ in the abelian group on $\epsilon^{-1}(\boldsymbol{h})$ determined by $*$, there are at most $m^{n-1}$ choices for extending $\varphi$ to all of $\epsilon^{-1}(\boldsymbol{h})$ when $\boldsymbol{h} \neq \boldsymbol{1}$. Hence

$$
\left|\mathscr{X}\left(\underline{\mathbf{D}}_{m}^{n}, \underline{\mathbf{D}}_{m}\right)\right| \leqslant 2^{n} \cdot m^{(n-1)\left(2^{n}-1\right)} m^{n}=2^{n} m^{(n-1) 2^{n}+1},
$$

as required.

PROPOSITION 5. ${\underset{\sim}{m}}_{m}$ is injective in $\mathscr{X}$.

PROOF. Let $\mathbf{X}$ be a closed substructure of ${\underset{\sim}{\mathbf{D}}}_{m}^{l}$ for some $I$, and let $\varphi \in \mathscr{X}\left(\mathbf{X}, \underline{\mathbf{D}}_{m}\right)$ be a continuous structure-preserving map. By Lemma 3 , the substructure $\mathbf{X}$ is closed under every endomorphism of $\underline{\mathbf{D}}_{m}$ and $\varphi$ preserves every endomorphism. In particular, $\mathbf{X}$ is closed under $\epsilon$ and $\varphi$ preserves $\epsilon$. We must find $\psi \in \mathscr{X}\left(\underline{\mathbf{D}}_{m}^{\prime}, \underline{\mathbf{D}}_{m}\right)$ with $\psi \uparrow_{\mathbf{X}}=\varphi$. On $\mathbf{D}_{m}^{l}$, the map $\epsilon$ is a continuous retraction onto $\{1, b\}^{I}$. Since $\mathbf{X}$ is closed under $\epsilon$, it follows easily that $\epsilon(X)=X \cap\{1, b\}^{I}$ and so is a closed subgroup of $\left\langle\{1, b\}^{I},+\right\rangle$. Thus $\varphi \Gamma_{\epsilon(X)}$ is a continuous +- homomorphism. By the Pontryagin duality for abelian groups of exponent 2 , there is a continuous +- homomorphism $\varphi_{1}:\{1, b\}^{I} \rightarrow\{1, b\}$ which extends $\varphi \uparrow_{\epsilon(X)}$.

The set

$$
\begin{aligned}
X^{\prime} & =X \cup \bigcup\left\{\alpha^{l}\left(\{1, b\}^{I}\right) \mid l \in \mathbb{Z}_{m}\right\} \\
& =X \cup \bigcup\left\{\left\{1, a^{\prime} b\right\}^{\prime} \mid l \in \mathbb{Z}_{m}\right\}
\end{aligned}
$$

is a closed substructure of ${\underset{\sim}{a_{m}}}_{m}^{I}$. (To see that $X^{\prime}$ is closed under $*$, use the fact that if $\boldsymbol{x} * \boldsymbol{y}$ is defined and $\boldsymbol{y} \in\left\{1, a^{l} b\right\}^{I}$, then $\boldsymbol{x} * \boldsymbol{y}=\alpha^{l}(\boldsymbol{x})$.) Define a map $\varphi_{2}: X^{\prime} \rightarrow D_{m}$ by

$$
\varphi_{2} \Upsilon_{\mathbf{x}}=\varphi, \quad \text { and } \quad \varphi_{2} \uparrow_{\left\{1, a^{\prime} b\right\}^{\prime}}=\alpha^{l} \circ \varphi_{1} \circ \alpha^{(m-l)} \quad \text { for all } l \in \mathbb{Z}_{m}
$$


For all $x \in X \cap\left\{1, a^{\prime} b\right\}$, we have

$$
\begin{aligned}
\alpha^{l} \circ \varphi_{1} \circ \alpha^{(m-l)}(\boldsymbol{x}) & =\alpha^{l} \varphi_{1}\left(\alpha^{(m-l)}(\boldsymbol{x})\right) & & \\
& =\alpha^{l} \varphi\left(\alpha^{(m-l)}(\boldsymbol{x})\right) & & \text { as } \alpha^{(m-l)}(\boldsymbol{x}) \in \epsilon(X) \text { and } \varphi_{1} \uparrow_{\epsilon(\mathbf{X})}=\varphi \\
& =\varphi \alpha^{l} \alpha^{(m-l)}(\boldsymbol{x}) & & \text { as } \varphi \text { preserves } \alpha \\
& =\varphi(\boldsymbol{x}), & &
\end{aligned}
$$

from which it follows that $\varphi_{2}$ is well-defined. Clearly, $\varphi_{2}$ is continuous and preserves the partial operation + and the constant 1 . We now show that $\varphi_{2}$ also preserves both the action of $\alpha$ and the partial operation $*$. If $x \in X$, then it is trivial that $\varphi_{2}$ preserves the action of $\alpha$ on $x$ since $\left.\varphi_{2}\right|_{\mathbf{X}}=\varphi$ and $\varphi$ preserves $\alpha$. If $\boldsymbol{x} \in\left\{1, a^{l} b\right\}^{\prime}$, then $\alpha(x) \in\left\{1, a^{l+1} b\right\}^{I}$ and hence

$$
\varphi_{2}(\alpha(x))=\alpha^{l+1} \varphi_{1} \alpha^{m-l-1}(\alpha(x))=\alpha\left(\alpha^{l} \varphi_{1} \alpha^{m-l}(x)\right)=\alpha\left(\varphi_{2}(x)\right),
$$

whence $\varphi_{2}$ preserves $\alpha$. Note that if $\boldsymbol{y} \in\left\{1, a^{l} b\right\}^{I}$, then $\varphi_{2}(\boldsymbol{y}) \in\left\{1, a^{l} b\right\}$ since $\varphi_{1}\left(\{1, b\}^{\prime}\right) \subseteq\{1, b\}$. If $\boldsymbol{x} \in X^{\prime}$ and $\boldsymbol{y} \in\left\{1, a^{l} b\right\}^{\prime}$ and $\boldsymbol{x} * \boldsymbol{y}$ is defined, then $\boldsymbol{x} * \boldsymbol{y}=\alpha^{l}(\boldsymbol{x})$ and hence

$$
\begin{aligned}
\varphi_{2}(\boldsymbol{x} * \boldsymbol{y}) & =\varphi_{2}\left(\alpha^{l}(\boldsymbol{x})\right) & & \\
& =\alpha^{l}\left(\varphi_{2}(\boldsymbol{x})\right) & & \text { as } \varphi_{2} \text { preserves } \alpha \\
& =\varphi_{2}(\boldsymbol{x}) * \varphi_{2}(\boldsymbol{y}) & & \text { as } \varphi_{2}(\boldsymbol{x}) \in\left\{1, a^{l} b\right\}^{l} .
\end{aligned}
$$

Hence $\varphi_{2}$ preserves $*$ and consequently $\varphi_{2} \in \mathscr{X}\left(\mathbf{X}^{\prime}, \mathbf{D}_{m}\right)$. That is, without loss of generality, we may assume that $X$ contains $\{1, b\}^{l}$ and thus

$$
Z:=\{1, b\}^{I} \cup\{1, a b\}^{I} \cup\left\{1, a^{2} b\right\}^{I} \cup \cdots \cup\left\{1, a^{m-1} b\right\}^{I} \subseteq X .
$$

Note that $\mathbf{Z}$ is a closed substructure of ${\underset{\mathbf{D}}{m}}_{m}^{I}$ and hence, by assumption, is a closed substructure of $\mathbf{X}$.

A simple-minded attempt to define the extension $\psi$ would proceed as follows. For any $\boldsymbol{h} \in\{1, b\}^{I}$, the set $X \cap \boldsymbol{\epsilon}^{-1}(\boldsymbol{h})$ is non-empty and so is a closed subgroup of $\epsilon^{-1}(\boldsymbol{h})=N^{l} \boldsymbol{h}$ (under the restriction of $*$ ). Now $\left\langle\epsilon^{-1}(\boldsymbol{h}) ; *\right\rangle$ is a compact topological abelian group of exponent $m$ and since $\varphi$ preserves $*$, the restriction $\varphi\left\lceil_{X \cap \epsilon^{-1}(h)}\right.$ is a continuous group homomorphism with codomain $\langle N \varphi(\boldsymbol{h}) ; *\rangle$. By the Pontryagin duality for abelian groups of exponent $m$, we can extend $\left.\varphi\right|_{X \cap \epsilon^{-1}(h)}$ to a continuous group homomorphism $\psi_{\boldsymbol{h}}: \epsilon^{-1}(\boldsymbol{h}) \rightarrow N \varphi(\boldsymbol{h})$. Doing this for each $\boldsymbol{h} \in\{1, b\}^{I}$, we obtain an extension of $\varphi$ to a map $\psi: D_{m}^{I} \rightarrow D_{m}$ given by $\left.\psi\right|_{\epsilon^{-1}(h)}=\psi_{h}$ for all $\boldsymbol{h} \in\{1, b\}^{I}$.

We claim that $\psi$ is structure preserving. Since $\mathbf{Z}$ is a substructure of $\mathbf{X}$, it is trivial that $\psi$ preserves both + and 1 and $\psi$ preserves $*$ by construction. That $\psi$ also preserves $\boldsymbol{\alpha}$ follows immediately once we have established the following lemma. 
LEMMA 6. Let $\mathbf{X}$ be a closed substructure of $\mathbf{D}_{m}^{I}$.

(a) For all $x \in D_{m}$ (and therefore for all $\left.\boldsymbol{x} \in X\right)$ we have $(x, \alpha(\epsilon(x))) \in \operatorname{dom}(*)$ and $\alpha(x)=x * \alpha(\epsilon(x))$.

(b) If $\psi: X \rightarrow D_{m}$ preserves $*$ and $\epsilon$ and $\psi \uparrow_{X \cap Z}$ preserves $\alpha$, then $\psi$ preserves $\alpha$.

(c) Let $\mathbf{Z}$ be a substructure of $\mathbf{X}$. If $\psi: X \rightarrow D_{m}$ preserves $*$ and $\psi \uparrow_{2}$ preserves $\alpha$, then $\psi$ preserves $\alpha$.

PROOF. The proof of (a) is a simple calculation and (b) follows easily from (a). Assume that $\mathbf{Z}$ is a substructure of $\mathbf{X}$, that $\psi$ preserves $*$ and that $\psi \uparrow_{Z}$ preserves $\alpha$. By (b), in order to establish (c) it remains to show that $\psi$ preserves $\epsilon$.

Since $Z \subseteq X$, for all $\boldsymbol{h} \in\{1, b\}^{\prime}$, the set $X \cap \epsilon^{-1}(\boldsymbol{h})$ is non-empty, whence $\left\langle X \cap \epsilon^{-1}(\boldsymbol{h}) ; *\right\rangle$ is a group with identity element $\boldsymbol{h}$. Since $\psi$ preserves $*$, we have $\psi(\boldsymbol{h}) \in\{1, b\}$ and $\psi\left(X \cap \epsilon^{-1}(\boldsymbol{h})\right) \subseteq N \psi(\boldsymbol{h})$. Thus

$$
\epsilon\left(\psi\left(X \cap \epsilon^{-1}(\boldsymbol{h})\right)\right) \subseteq \epsilon(N \psi(\boldsymbol{h}))=\{\psi(\boldsymbol{h})\},
$$

which gives $\epsilon(\psi(\boldsymbol{x}))=\psi(\epsilon(\boldsymbol{x}))$ for all $\boldsymbol{x} \in X$, as required.

Unfortunately, if $I$ is infinite, we cannot guarantee that the extension $\psi$ is continuous and the simple-minded approach falters. Nevertheless, the basic idea can be salvaged. Obviously, we need to invoke some kind of compactness argument. The following lemma plays a crucial role.

LEMMA 7: THE GOOD, THE BAD, BUT NO UGLY. Let $A$ and I be sets with A finite. Suppose that, for every finite $I^{\prime} \subseteq I$, each element of $A^{I^{\prime}}$ is labeled either 'good' or 'bad' and that if $I^{\prime \prime} \subseteq I^{\prime}$ and $\boldsymbol{x} \in A^{I^{\prime}}$ is bad, then so is $\left.\boldsymbol{x}\right|_{I^{\prime \prime}} \in A^{I^{\prime \prime}}$. Then either there is a finite $I^{\prime} \subseteq I$ such that each element of $A^{I^{\prime}}$ is good, or there is an $x \in A^{\prime}$ such that $\boldsymbol{x} \uparrow_{I^{\prime}}$, is bad for each finite $I^{\prime} \subseteq I$.

Proof. Endow $A$ with the discrete topology and $A^{l}$ with the product topology; then $A^{I}$ is a compact space with a basis of clopen sets. For finite $I^{\prime} \subseteq I$, let $X\left(I^{\prime}\right):=\left\{x \in A^{I}|\boldsymbol{x}|_{I^{\prime}}\right.$ is bad $\}$; it is a closed set. Then by the finite intersection property, either $\bigcap\left\{X\left(I^{\prime}\right) \mid I^{\prime} \subseteq I\right.$ is finite $\}$ is non-empty or there are finitely many finite sets $I_{1}, \ldots, I_{k}$ such that $X\left(I_{1}\right) \cap \cdots \cap X\left(I_{k}\right)$ is empty. In the former case, take $x \in \bigcap\left\{X\left(I^{\prime}\right) \mid I^{\prime} \subseteq I\right.$ is finite $\}$; then $\left.x\right|_{I^{\prime}}$ is bad for any finite $I^{\prime} \subseteq I$. In the latter case, let $I^{\prime}=I_{1} \cup \cdots \cup I_{k}$; then, as $X\left(I^{\prime}\right) \subseteq X\left(I_{j}\right)$ for $1 \leqslant j \leqslant k$, the set $X\left(I^{\prime}\right)$ is empty and so every member of $A^{I^{\prime}}$ is good.

Let us apply the Good, the Bad, but no Ugly Lemma to $\{1, b\}^{I}$. For $\boldsymbol{h} \in\{1, b\}^{I}$ and finite $I^{\prime} \subseteq I$, define

$$
\Gamma_{I^{\prime}}^{h}:=\left\{\left(\boldsymbol { x } \lceil _ { I ^ { \prime } } , \varphi ( \boldsymbol { x } ) ) | \boldsymbol { x } \in X \text { and } \epsilon \left(\boldsymbol{x}\left\lceil_{I^{\prime}}\right)=\boldsymbol{h}\left\lceil_{l^{\prime}}\right\}\right.\right.\right.
$$


Call $\boldsymbol{h} \Gamma_{I^{\prime}}$ 'good' if $\Gamma_{I^{\prime}}^{h}$ is a subset of the graph of a $*$-preserving map defined on $\epsilon^{-1}\left(\boldsymbol{h} \uparrow_{I^{\prime}}\right)=\epsilon^{-1}(\boldsymbol{h}) \uparrow_{I^{\prime}}$; otherwise, call $\left.\boldsymbol{h}\right|_{I^{\prime}}$ 'bad'. Let $I^{\prime \prime} \subseteq I^{\prime}$ and let $\pi$ denote the natural restriction map from $\left.\epsilon^{-1}(\boldsymbol{h})\right|_{l^{\prime}}$ to $\left.\epsilon^{-1}(\boldsymbol{h})\right|_{l^{\prime \prime}}$. If $\gamma$ is an extension of $\Gamma_{l^{\prime \prime}}^{h}$ to a *-preserving map on $\epsilon^{-1}\left(\boldsymbol{h}\left\lceil_{I^{\prime \prime}}\right)\right.$, then $\gamma \circ \pi$ is an extension of $\Gamma_{I^{\prime}}^{h}$ to a $*$-preserving map on $\epsilon^{-1}\left(\left.\boldsymbol{h}\right|_{l^{\prime}}\right)$. Hence 'badness' is hereditary in the sense required by the lemma. Thus, by the lemma, either

(a) there is a finite subset $I^{\prime}$ of $I$ such that every member $\boldsymbol{h}^{\prime}$ of $\{1, b\}^{I^{\prime}}$ is good, or

(b) there exists $\boldsymbol{h} \in\{1, b\}^{I}$ such that $\boldsymbol{h}_{I^{\prime}}$ is bad, for all finite $I^{\prime} \subseteq I$.

Case (a). For each $\boldsymbol{h}^{\prime} \in\{1, b\}^{I^{\prime}}$, let $\psi_{\boldsymbol{h}^{\prime}}: \epsilon^{-1}\left(\boldsymbol{h}^{\prime}\right) \rightarrow D_{m}$ be a $*$-preserving map which satisfies $\psi_{\boldsymbol{h}^{\prime}}\left(\left.\boldsymbol{x}\right|_{I^{\prime}}\right)=\varphi(\boldsymbol{x})$ for all $\boldsymbol{x} \in X$ with $\epsilon\left(\left.\boldsymbol{x}\right|_{I^{\prime}}\right)=\boldsymbol{h}^{\prime}$, and define $\psi^{\prime}: D_{m}^{I^{\prime}} \rightarrow D_{m}$ to be the union of the maps $\psi_{\boldsymbol{h}^{\prime}}$ for $\boldsymbol{h}^{\prime} \in\{1, b\}^{I^{\prime}}$. We claim that $\psi^{\prime}$ is an $\mathscr{X}$-morphism. Let $\boldsymbol{x}_{1}^{\prime}, \boldsymbol{x}_{2}^{\prime} \in\{1, b\}^{I^{\prime}}=\operatorname{dom}_{\mathbf{Q}_{m}^{\prime \prime}}(+)$. Thus as $Z \subseteq X$, there exist $\boldsymbol{x}_{1}, \boldsymbol{x}_{2} \in\{1, b\}^{l} \subseteq X$ with $x_{i} \uparrow_{I^{\prime}}=x_{i}^{\prime}$. Thus $\left(x_{1}, x_{2}\right) \in \operatorname{dom}_{\mathbf{X}}(+)$ and so $x_{1}+x_{2} \in X$ and $\left(x_{1}+x_{2}\right) \uparrow_{I^{\prime}}=x_{1}^{\prime}+x_{2}^{\prime}$. Define $h^{\prime}:=\epsilon\left(x_{1}+x_{2}\right)$ and $h_{i}^{\prime}:=\epsilon\left(x_{i}^{\prime}\right)$. Thus

$$
\begin{aligned}
\psi^{\prime}\left(\boldsymbol{x}_{1}^{\prime}+\boldsymbol{x}_{2}^{\prime}\right) & =\psi_{\boldsymbol{h}^{\prime}}\left(\boldsymbol{x}_{1}^{\prime}+\boldsymbol{x}_{2}^{\prime}\right) & & \left(\text { definition of } \psi^{\prime}\right) \\
& =\psi_{\boldsymbol{h}^{\prime}}\left(\left(\boldsymbol{x}_{1}+\boldsymbol{x}_{2}\right) \uparrow_{l^{\prime}}\right) & & \\
& =\varphi\left(\boldsymbol{x}_{1}+\boldsymbol{x}_{2}\right) & & \text { (definition of } \left.\psi_{\boldsymbol{h}^{\prime}}\right) \\
& =\varphi\left(\boldsymbol{x}_{1}\right)+\varphi\left(\boldsymbol{x}_{2}\right) & & \text { (as } \varphi \text { preserves }+ \text { ) } \\
& =\psi_{\boldsymbol{h}_{1}^{\prime}}\left(\boldsymbol{x}_{1} \uparrow_{l^{\prime}}\right)+\psi_{\boldsymbol{h}_{2}^{\prime}}\left(\boldsymbol{x}_{2} \uparrow_{l^{\prime}}\right) & & \left(\text { definition of } \psi_{\boldsymbol{h}_{i}^{\prime}}\right) \\
& =\psi_{\boldsymbol{h}_{1}^{\prime}}\left(\boldsymbol{x}_{1}^{\prime}\right)+\psi_{\boldsymbol{h}_{2}^{\prime}}\left(\boldsymbol{x}_{2}^{\prime}\right) & & \\
& =\psi^{\prime}\left(\boldsymbol{x}_{1}^{\prime}\right)+\psi^{\prime}\left(\boldsymbol{x}_{2}^{\prime}\right), & &
\end{aligned}
$$

whence $\psi^{\prime}$ preserves + . By Lemma 6 applied to $\psi^{\prime}$, it remains to show that $\left.\psi^{\prime}\right|_{\mathbf{Z}^{\prime}}$ preserves $\alpha$, where $Z^{\prime}:=\bigcup\left\{\left\{1, a^{\prime} b\right\}^{I^{\prime}} \mid l \in \mathbb{Z}_{m}\right\}$. Let $\boldsymbol{x}^{\prime} \in Z^{\prime}$ and define $h^{\prime}:=$ $\epsilon\left(x^{\prime}\right)=\epsilon\left(\alpha\left(x^{\prime}\right)\right)$. Let $x \in Z$ with $\left.x\right|_{I^{\prime}}=x^{\prime}$. Note that $x \in X$ as $Z \subseteq X$. Now

$$
\begin{aligned}
\psi^{\prime}\left(\alpha\left(\boldsymbol{x}^{\prime}\right)\right) & =\psi_{\boldsymbol{h}^{\prime}}\left(\left.\alpha(\boldsymbol{x})\right|_{l^{\prime}}\right) & & \left(\text { definition of } \psi^{\prime}\right) \\
& =\varphi(\alpha(\boldsymbol{x})) & & \left(\text { definition of } \psi_{h^{\prime}}\right) \\
& =\alpha(\varphi(\boldsymbol{x})) & & (\text { as } \varphi \text { preserves } \alpha) \\
& =\alpha\left(\psi_{h^{\prime}}\left(\left.\boldsymbol{x}\right|_{I^{\prime}}\right)\right) & & \left(\text { definition of } \psi_{\boldsymbol{h}^{\prime}}\right) \\
& =\alpha\left(\psi_{\boldsymbol{h}^{\prime}}\left(\boldsymbol{x}^{\prime}\right)\right) & & \\
& =\alpha\left(\psi^{\prime}\left(\boldsymbol{x}^{\prime}\right)\right), & &
\end{aligned}
$$

and consequently $\psi^{\prime}$ preserves $\alpha$ on $\mathbf{Z}^{\prime}$, as required. Thus $\psi^{\prime}:{\underset{\sim}{m}}_{m}^{I^{\prime}} \rightarrow \underline{\sim}_{m}$ is an $\mathscr{X}$-morphism, as claimed.

Finally, let $\pi_{l^{\prime}}: \underline{\mathbf{D}}_{m}^{l} \rightarrow\left(\mathbf{D}_{m}^{l^{\prime}}\right)$ denote the restriction map. Then the map $\psi^{\prime} \circ$ $\pi_{I^{\prime}}: \underline{\mathbf{D}}_{m}^{I} \rightarrow \underline{\mathbf{D}}_{m}$ is an $\mathscr{X}$-morphism which extends $\varphi$ since $\psi(\boldsymbol{x})=\psi^{\prime}\left(\left.\boldsymbol{x}\right|_{I^{\prime}}\right)=$ $\psi_{\epsilon\left(x_{\left.I^{\prime}\right)}\right)}\left(\left.\boldsymbol{x}\right|_{I^{\prime}}\right)=\varphi(\boldsymbol{x})$ for all $\boldsymbol{x} \in X$. 
Case (b). Assume that $\boldsymbol{h} \in\{1, b\}^{I}$ with $\left.\boldsymbol{h}\right|_{I^{\prime}}$ bad, for all finite $I^{\prime} \subseteq I$, that is, for every finite subset $I^{\prime}$ of $I$, the set

$$
\Gamma_{I^{\prime}}^{\boldsymbol{h}}:=\left\{\left(\left.\boldsymbol{x}\right|_{I^{\prime}}, \varphi(\boldsymbol{x})\right) \mid \boldsymbol{x} \in X \text { and } \epsilon\left(\left.\boldsymbol{x}\right|_{I^{\prime}}\right)=\left.\boldsymbol{h}\right|_{I^{\prime}}\right\} .
$$

is not a subset of a $*$-preserving map defined on $\epsilon^{-1}\left(\boldsymbol{h} \Gamma_{I^{\prime}}\right)$. Define $X^{0}:=X \cup \epsilon^{-1}(\boldsymbol{h})$. Then $X^{0}$ is closed under $*$ and, as in the simple-minded approach, we may apply the Pontryagin duality for abelian groups of exponent $m$ to extend $\varphi$ to a continuous *-preserving map $\varphi^{0}: X^{0} \rightarrow D_{m}$. Since $\epsilon\left(\left.\boldsymbol{x}\right|_{l^{\prime}}\right)=\left.\epsilon(\boldsymbol{x})\right|_{\ell^{\prime}}$ and since $\epsilon^{-1}(\boldsymbol{h}) \subseteq X^{0}$, we have

$$
\begin{aligned}
\Gamma_{I^{\prime}}^{\boldsymbol{h}} & =\left\{\left(\left.\boldsymbol{x}\right|_{I^{\prime}}, \varphi(\boldsymbol{x})\right) \mid \boldsymbol{x} \in X \text { and }\left.\epsilon(\boldsymbol{x})\right|_{I^{\prime}}=\boldsymbol{h} \uparrow_{I^{\prime}}\right\} \\
& \subseteq\left\{\left(\left.\boldsymbol{x}\right|_{I^{\prime}}, \varphi^{0}(\boldsymbol{x})\right) \mid \boldsymbol{x} \in X \text { and }\left.\epsilon(\boldsymbol{x})\right|_{I^{\prime}}=\left.\boldsymbol{h}\right|_{I^{\prime}}\right\} \\
& =\left\{\left(\left.\boldsymbol{x}\right|_{I^{\prime}}, \varphi^{0}(\boldsymbol{x})\right) \mid \boldsymbol{x} \in \epsilon^{-1}(\boldsymbol{h})\right\} .
\end{aligned}
$$

The set $\Gamma^{0}:=\left\{\left(\left.\boldsymbol{x}\right|_{I^{\prime}}, \varphi^{0}(\boldsymbol{x})\right) \mid \boldsymbol{x} \in \boldsymbol{\epsilon}^{-1}(\boldsymbol{h})\right\}$ is easily seen to be a $*$-closed subset of $D_{m}^{I^{\prime}} \times D_{m}$. Hence, if $\Gamma_{0}$ were the graph of a map, then it would be the graph of a $*$-preserving map defined on $\epsilon^{-1}(\boldsymbol{h}) \uparrow_{l^{\prime}}$, contradicting the fact that $\boldsymbol{h} \uparrow_{l^{\prime}}$ is bad. Thus there exist $\boldsymbol{y}, \boldsymbol{z} \in X$ (depending on $I^{\prime}$ ) such that $\left.\boldsymbol{y}\right|_{I^{\prime}}=\left.\boldsymbol{z}\right|_{I^{\prime}}$ but $\varphi^{0}(\boldsymbol{y}) \neq \varphi^{0}(z)$. This means that the continuous map $\varphi^{0}$ does not depend on any finite subset of $I$, a contradiction to the fact that every continuous map from a closed subspace of any power of ${\underset{\sim}{\mathbf{D}}}_{m}$ into ${\underset{\mathbf{D}}{m}}_{m}$ depends on only finitely many components. Hence, Case (b) cannot occur.

PROPOSITION 8. If $\mathbf{X}$ is a closed substructure of $\mathbf{D}_{m}^{I}$ for some set $I$ and $\boldsymbol{y} \in D_{m}^{I} \backslash X$, then there exists a continuous morphism $\psi:{\underset{\sim}{\mathbf{D}}}_{m}^{I} \rightarrow \mathbf{D}_{m}$ such that $\psi \uparrow_{X}=\underline{\mathbf{1}}$ but $\psi(y) \neq 1$, where 1 is the constant map onto 1 .

PROOF. Let $\mathbf{X}$ be a substructure of $\underset{\sim}{\mathbf{D}_{m}^{l}}$ and let $\boldsymbol{y} \notin X$.

If $y \in Z$, say $y \in\left\{1, a^{l} b\right\}^{I}$, then define $\boldsymbol{y}_{1} \in\{1, b\}^{l}$ by

$$
\boldsymbol{y}_{1}(i)= \begin{cases}1 & \text { if } \boldsymbol{y}(i)=1, \\ b & \text { if } \boldsymbol{y}(i)=a^{\prime} b .\end{cases}
$$

Note that $\alpha^{\prime}\left(\boldsymbol{y}_{1}\right)=\boldsymbol{y}$ whence $\boldsymbol{y}_{1} \in\{1, b\}^{I} \backslash X$ (as $X$ is closed under $\alpha$ ). Since the Pontryagin duality for $\mathscr{A}_{2}$ is as strong, there exists a continuous group homomorphism $\varphi_{1}:\{1, b\}^{I} \rightarrow\{1, b\}$ such that $\varphi_{1} \uparrow_{\epsilon(\mathbf{X})}=\underline{1}$ but $\varphi_{1}\left(\boldsymbol{y}_{1}\right) \neq 1$. As in the proof of Proposition 5, there exists an extension $\psi:{\underset{\sim}{\mathbf{D}}}_{m}^{I} \rightarrow \underline{\mathbf{D}}_{m}$ with $\psi \Gamma_{\mathbf{X}}=\underline{\mathbf{1}}$ and $\psi \uparrow_{\{1, b\}^{\prime}}=\varphi_{1}$. Suppose that $\psi(\boldsymbol{y})=1$; then

$$
\varphi_{1}\left(y_{1}\right)=\psi\left(y_{1}\right)=\psi\left(\alpha^{m-l}(y)\right)=\alpha^{m-l}(\psi(y))=\alpha^{m-l}(1)=1,
$$


a contradiction. Hence $\psi(y) \neq 1$, as required.

If $y \notin Z$, then, as in the proof of Proposition 5, we may assume that $Z \subseteq X$. Since $\mathbf{X}$ is a closed subspace of $\mathbf{D}_{m}^{I}$ and $\boldsymbol{y} \notin X$, there exists a finite subset $I^{\prime}$ of $I$ such that $\boldsymbol{x} \uparrow_{I^{\prime}} \neq \boldsymbol{y} \uparrow_{I^{\prime}}$ for all $\boldsymbol{x} \in X$. Let $\boldsymbol{h}:=\epsilon(\boldsymbol{y}), \boldsymbol{y}^{\prime}:=\left.\boldsymbol{y}\right|_{I^{\prime}}$ and $\boldsymbol{h}^{\prime}:=\boldsymbol{h} \uparrow_{I^{\prime}}=\epsilon\left(\boldsymbol{y}^{\prime}\right)$. Since the Pontryagin duality for $\mathscr{A}_{m}$ is strong, there exists a $*$-preserving map $\psi_{\boldsymbol{h}^{\prime}}: \epsilon^{-1}\left(\boldsymbol{h}^{\prime}\right) \rightarrow$ $\mathbf{D}_{m}$ such that $\left.\psi_{\boldsymbol{h}^{\prime}}(\boldsymbol{x}\rceil_{I^{\prime}}\right)=1$ for all $\boldsymbol{x} \in X$ with $\left.\epsilon(\boldsymbol{x}\rceil_{I^{\prime}}\right)=\boldsymbol{h}^{\prime}$ while $\psi_{h^{\prime}}\left(\left.\boldsymbol{y}\right|_{I^{\prime}}\right) \neq 1$. Define $\psi^{\prime}: D_{m}^{I^{\prime}} \rightarrow D_{m}$ by

$$
\psi^{\prime}(z)= \begin{cases}1 & \text { if } z \notin \epsilon^{-1}\left(\boldsymbol{h}^{\prime}\right), \\ \psi_{\boldsymbol{h}^{\prime}}(z) & \text { if } z \in \epsilon^{-1}\left(\boldsymbol{h}^{\prime}\right),\end{cases}
$$

and let $\psi:=\psi^{\prime} \circ \pi_{I^{\prime}}$. Clearly, $\psi:{\underset{\sim}{\mathbf{D}}}_{m}^{I} \rightarrow{\underset{\sim}{\mathbf{D}}}_{m}$ preserves $*$ and $\psi \uparrow_{\mathbf{z}}$ preserves $\alpha$ (since $Z \subseteq X$ and $\psi(X)=\{1\})$. Thus, Lemma 6(c), with $\mathbf{X}=\mathbf{D}_{m}^{\prime}$, implies that $\psi$ preserves $\alpha$. Finally, $\{1, b\}^{\prime} \subseteq Z \subseteq X$ implies that $\psi$ takes the constant value 1 on $\{1, b\}$ and so preserves + . Thus $\psi$ is the required $\mathscr{X}$-morphism.

This concludes the proof of Theorem 2. We close the paper with an interesting special case of the problem stated in the introduction.

PROBLEM. Does every finite metacyclic group admit a duality? In particular, does every dihedral group of order $2 m$, with $m$ even, admit a duality. Indeed, does $\underline{\mathbf{D}}_{4}$ admit a duality?

NOTE ADDED IN PROOF. Cs. Szabo and the second author have shown that no finite, non-abelian nilpotent group admits a duality.

\section{References}

[1] D. M. Clark and B. A. Davey, 'The quest for strong dualities', J. Austral. Math. Soc. (Series A) 58 (1995), 248-280.

[2] _ 'When is a natural duality 'good'?', Algebra Universalis 35 (1996), 237-267.

[3] — - 'Natural dualities for the working algebraist', in preparation.

[4] B. A. Davey, 'Duality theory on ten dollars a day', in: Algebras and orders (I. G. Rosenberg and G. Sabidussi, eds) NATO Advanced Study Institute Series, Series C, Vol. 389 (Kluwer, 1993) pp. 71-111.

[5] — 'Dualizability of finite abelian groups and some other finite algebras', preprint, 1994.

[6] B. A. Davey and H. Werner, 'Dualities and equivalences for varieties of algebras', in: Contributions to lattice theory (Szeged, 1980), (A. P. Huhn and E. T. Schmidt, eds.) Colloq. Math. Soc. János Bolyai, Vol. 33 (North-Holland, Amsterdam, 1983) pp. 101-275.

[7] L. G. Kovács, 'Free groups in a dihedral variety', Proc. Roy. Irish Acad. 89A (1989), 115-117.

[8] H. Neumann, Varieties of groups, Ergebnisse der Mathematik und ihrer Grenzgebeite 37 (Springer, Berlin, 1967). 
La Trobe University

Bundoora VIC 3083

Australia

e-mail: davey@latcs1.lat.oz.au
University of Manitoba

Winnipeg Manitoba

Canada R3T 2N2

e-mail: qbush@ccu.umanitoba.ca 